

\title{
Stability Analysis for Neutral Systems with Mixed Delays
}

\author{
${ }^{*}$ Xin-Ge Liu ${ }^{a, b, c}$, Min $\mathrm{Wu}^{b}$, Ralph Martin ${ }^{c}$, Mei-Lan Tang ${ }^{a}$ \\ ${ }^{a}$ School of Mathematical Science and Computing Technology, Central South University \\ Changsha, Hunan 410083, China \\ ${ }^{b}$ School of Information Science and Engineering, Central South University \\ Changsha, Hunan 410083, China \\ ${ }^{c}$ School of Computer Science, Cardiff University, Cardiff, UK
}

\begin{abstract}
Relationships between system states contained in the neutral equation are used to address the delay-dependent stability of a neutral system with time-varying state delay. Using linear matrix inequalities, we present a new asymptotic stability criterion, and a new robust stability criterion, for neutral systems with mixed delays. Since the criteria take into account the sizes of the neutral delay, discrete delay and the derivative of discrete delay, they are less conservative than those produced by previous approaches. Numerical examples are presented to demonstrate that these criteria are indeed more effective.
\end{abstract}

Keywords: Neutral system; Time-varying discrete delay; Neutral delay; Linear matrix inequality.

\section{Introduction}

A neutral time delay system contains delays both in its state, and in its derivatives of state. Such systems are often encountered in engineering (e.g. in heat exchanger analysis), and in biology (e.g. in population ecology). Various modern control technologies, like repetitive control, use neutral systems via the insertion of an artificial neutral delay into a control loop, in order to boost control performance for systems with periodic signals [8]. Studies of delay-dependent stability criteria for neutral systems have focused mainly on cases with identical delays in neutral and discrete terms: see, for example $[1,2,6,12,13,16]$. Other papers have presented criteria that depend only on the size of the discrete delays, and not on the size of the neutral delays: see, for example, [5, 10, 15, 17].

Recently, He et al [9] presented a new delay-dependent stability criterion for neutral systems with mixed delays, i.e. where the discrete delay and neutral delay are different constants. In order to obtain their criteria, the relation between the state $x(t-\tau)$ and $x(t)-\int_{t-\tau}^{t} \dot{x}(s) d s$ is taken into account in the derivative of a Lyapunov-Krasovskii functional through the Leibniz-Newton formula. Obviously, however, this stability criterion can not be applied to neutral systems with time-varying discrete delay. Their stability criterion does not take into account the information contained in the system equation, and so their stability conditions have a conservatism which can be improved upon.

The contribution of this paper is to make use of the information contained in the system equation to investigate stability criteria for neutral systems with mixed delays. Initially, we consider

*Corresponding Author Tel:+86-731-8830812; fax:+86-731-2656571. E-mail address: liuxgliuhua@163.com 
the nominal neutral system:

$$
\dot{x}(t)-C \dot{x}(t-h)=A x(t)+A_{1} x(t-\tau(t)) .
$$

This equation implies that

$$
x(t)-C x(t-h)-x(t-\tau(t))+C x(t-\tau(t)-h)=\int_{t-\tau(t)}^{t} A x(s)+A_{1} x(s-\tau(s)) d s
$$

and

$$
x(t)-(C+I) x(t-h)+C x(t-2 h)=\int_{t-h}^{t} A x(s)+A_{1} x(s-\tau(s)) d s .
$$

These two equations motivate us to consider relationships between the state vectors $x(t), x(t-\tau(t))$, $x(t-\tau(t)-h), x(t-h), x(t-2 h)$, and the derivative of the state vector at $x(t-h)$, i.e. $\dot{x}(t-h)$. The relationships between these state vectors and the derivative of the state vector can be expressed using suitable matrices whose entries can be chosen. Based on these relations and the LeibnizNewton formula, a new Lyapunov functional is introduced. A new, less conservative stability criterion for such a nominal neutral system is derived without the use of inequalities (such as those due to Moon [14]) to bound the time derivative of the Lyapunov functional.

Furthermore, this criterion is both neutral delay dependent and discrete delay dependent, and at the same time, is dependent on the derivative of the discrete delay. This criterion can easily be extended to a neutral system with time-varying uncertainties.

Finally, we give numerical examples to demonstrate that our proposed criteria significantly improve the allowed maximum upper bounds for the delay compared to existing results.

For simplicity, in the rest of the paper, in symmetric block matrices or long matrix expressions, we use $*$ to represent some term that is induced by symmetry.

\section{System description and main results}

In this section, we establish new stability criteria for a neutral system with mixed delays.

Consider the following neutral system with time-varying delay in the state:

$$
\begin{aligned}
\dot{x}(t)-C \dot{x}(t-h) & =(A+\Delta A(t)) x(t)+\left(A_{1}+\Delta A_{1}(t)\right) x(t-\tau(t)), \\
x(t) & =\phi(t), \quad t \in[-H, 0],
\end{aligned}
$$

where $x(t) \in R^{n}$ is the state vector, and $C$ is a constant matrix. $A$ and $A_{1}$ represent the fixed parts, and $\Delta A(t)$ and $\Delta A_{1}(t)$ the time-varying unknown parts, respectively, of the system matrices.

The spectral radius of the matrix $C, \rho(C)$, must satisfy $\rho(C)<1$. The time delay $\tau(t)$ is a time-varying continuous function satisfying

$$
0 \leq \tau(t) \leq \tau, \quad \dot{\tau}(t) \leq \mu<1,
$$

where $\tau$ and $\mu$ are constants. $H$ is defined by $H=\max (\tau, h) . \phi(t)$ is a continuous vector-valued function of $t \in[-H, 0]$. The time-varying uncertainties are of the form

$$
\left[\Delta A(t), \Delta A_{1}(t)\right]=D F(t)\left[E, E_{1}\right]
$$

where $D, E$, and $E_{1}$ are constant matrices of appropriate dimensions. $F(t)$ is an unknown and possibly time-varying real matrix with Lebesgue measurable elements and with Euclidean norm satisfying

$$
\|F(t)\| \leq 1, \quad \forall t
$$

We start by considering the nominal system associated with the system in Equation (1):

$$
\begin{aligned}
\dot{x}(t)-C \dot{x}(t-h) & =A x(t)+A_{1} x(t-\tau(t)), \\
x(t) & =\phi(t), \quad t \in[-H, 0] .
\end{aligned}
$$


In order to simplify the treatment of the problem, we define the operator $G$ and the function $\xi(t)$ as follows:

$$
G x_{t}=x(t)-C x(t-h)
$$

and

$$
\xi(t)=A x(t)+A_{1} x(t-\tau(t)) .
$$

Definition 1. The operator $G$ is said to be stable if the solution of the homogeneous difference equation

$$
G x_{t}=0, \quad t \geq 0, \quad x_{0}=\psi \in\{\phi \in C[-h, 0]: G \phi=0\}
$$

is uniformly asymptotically stable.

In the rest of this section, a new delay-dependent stability criterion for the nominal neutral system given in Equation (5) will be presented and its correctness proved.

Theorem 1. For given scalars $h>0, \tau>0$, and $\mu$, the neutral system in Equation (5) is asymptotically stable for any delay $\tau(t)$ satisfying Condition (2) if the operator $G$ is stable, and there exist positive definite matrices $P>0, P_{1}>0, Q_{1}>0, Q_{2}>0, Q_{3}>0$, and $R_{k}>0, S_{k}>0$, $Z_{k}>0, k=1,2$, and appropriately dimensioned matrices $T_{i}, N_{i}, Y_{i}, W_{i}, i=1, \ldots, 6$ such that the following symmetric linear matrix inequality (LMI) holds:

$$
\left[\begin{array}{cccccccccccc}
\tilde{\Omega}_{11} & \tilde{\Omega}_{12} & \tilde{\Omega}_{13} & \tilde{\Omega}_{14} & \tilde{\Omega}_{15} & \tilde{\Omega}_{16} & -\tau T_{1} & -\tau N_{1} & -h Y_{1} & -h W_{1} & A^{T} M & A^{T} S \\
* & \tilde{\Omega}_{22} & \tilde{\Omega}_{23} & \tilde{\Omega}_{24} & \tilde{\Omega}_{25} & \tilde{\Omega}_{26} & -\tau T_{2} & -\tau N_{2} & -h Y_{2} & -h W_{2} & A_{1}^{T} M & A_{1}^{T} S \\
* & * & \tilde{\Omega}_{33} & \tilde{\Omega}_{34} & \tilde{\Omega}_{35} & \tilde{\Omega}_{36} & -\tau T_{3} & -\tau N_{3} & -h Y_{3} & -h W_{3} & 0 & 0 \\
* & * & * & \tilde{\Omega}_{44} & \tilde{\Omega}_{45} & \tilde{\Omega}_{46} & -\tau T_{4} & -\tau N_{4} & -h Y_{4} & -h W_{4} & 0 & 0 \\
* & * & * & * & \tilde{\Omega}_{55} & \tilde{\Omega}_{56} & -\tau T_{5} & -\tau N_{5} & -h Y_{5} & -h W_{5} & 0 & 0 \\
* & * & * & * & * & \tilde{\Omega}_{66} & -\tau T_{6} & -\tau N_{6} & -h Y_{6} & -h W_{6} & 0 & C^{T} S \\
* & * & * & * & * & * & -\tau R_{1} & 0 & 0 & 0 & 0 & 0 \\
* & * & * & * & * & * & * & -\tau R_{2} & 0 & 0 & 0 & 0 \\
* & * & * & * & * & * & * & * & -h Z_{1} & 0 & 0 & 0 \\
* & * & * & * & * & * & * & * & * & -h Z_{2} & 0 & 0 \\
* & * & * & * & * & * & * & * & * & * & -M & 0 \\
* & * & * & * & * & * & * & * & * & * & * & -S
\end{array}\right]<0
$$

where

$$
\begin{aligned}
\tilde{\Omega}_{11}= & P A+A^{T} P+P_{1} A+A^{T} P_{1}+Q_{1}+S_{1}+S_{2}+ \\
& Y_{1}+Y_{1}^{T}+T_{1}+T_{1}^{T}+W_{1}+W_{1}^{T}+N_{1}+N_{1}^{T}, \\
\tilde{\Omega}_{12}= & P A_{1}+P_{1} A_{1}+Y_{2}^{T}-T_{1}+T_{2}^{T}+W_{2}^{T}-N_{1}+N_{2}^{T}, \\
\tilde{\Omega}_{13}= & Y_{3}^{T}+T_{1} C+T_{3}^{T}+W_{3}^{T}+N_{3}^{T}, \\
\tilde{\Omega}_{14}= & -A^{T} P_{1} C-Y_{1}(C+I)+Y_{4}^{T}-T_{1} C+T_{4}^{T}-W_{1}+W_{4}^{T}+N_{4}^{T}, \\
\tilde{\Omega}_{15}= & Y_{1} C+Y_{5}^{T}+T_{5}^{T}+W_{5}^{T}+N_{5}^{T}, \\
\tilde{\Omega}_{16}= & P C+Y_{6}^{T}+T_{6}^{T}+W_{6}^{T}+N_{6}^{T}, \\
\tilde{\Omega}_{22}= & -(1-\mu) S_{1}-T_{2}-T_{2}^{T}-N_{2}-N_{2}^{T}, \\
\tilde{\Omega}_{23}= & T_{2} C-T_{3}^{T}-N_{3}^{T}, \\
\tilde{\Omega}_{24}= & -A_{1}^{T} P_{1} C-Y_{2}(C+I)-T_{2} C-T_{4}^{T}-W_{2}-N_{4}^{T}, \\
\tilde{\Omega}_{25}= & Y_{2} C-T_{5}^{T}-N_{5}^{T}, \\
\tilde{\Omega}_{26}= & -T_{6}^{T}-N_{6}^{T}, \\
\tilde{\Omega}_{33}= & -(1-\mu) S_{2}+T_{3} C+C^{T} T_{3}^{T}, \\
\tilde{\Omega}_{34}= & -Y_{3}(C+I)-T_{3} C+C^{T} T_{4}^{T}-W_{3}, \\
\tilde{\Omega}_{35}= & Y_{3} C+C^{T} T_{5}^{T}, \\
\tilde{\Omega}_{36}= & C^{T} T_{6}^{T},
\end{aligned}
$$




$$
\begin{aligned}
\tilde{\Omega}_{44} & =-Q_{1}+Q_{2}-Y_{4}(C+I)-(C+I)^{T} Y_{4}^{T}-T_{4} C-C^{T} T_{4}^{T}-W_{4}-W_{4}^{T}, \\
\tilde{\Omega}_{45} & =Y_{4} C-(C+I)^{T} Y_{5}^{T}-C^{T} T_{5}^{T}-W_{5}^{T} \\
\tilde{\Omega}_{46} & =-(C+I)^{T} Y_{6}^{T}-C^{T} T_{6}^{T}-W_{6}^{T} \\
\tilde{\Omega}_{55} & =-Q_{2}+Y_{5} C+C^{T} Y_{5}^{T} \\
\tilde{\Omega}_{56} & =C^{T} Y_{6}^{T} \\
\tilde{\Omega}_{66} & =-Q_{3} \\
M & =h Z_{1}+\tau R_{1} \\
S & =Q_{3}+h Z_{2}+\tau R_{2} .
\end{aligned}
$$

Proof. Choose the Lyapunov functional candidate for the system with time-varying state delay given in Equation (5) to be:

$$
\begin{aligned}
V\left(x_{t}\right) & =V_{1}\left(x_{t}\right)+V_{2}\left(x_{t}\right)+V_{3}\left(x_{t}\right)+V_{4}\left(x_{t}\right)+V_{5}\left(x_{t}\right)+V_{6}\left(x_{t}\right) ; \\
V_{1}\left(x_{t}\right) & =x^{T}(t) P x(t)+\left(G x_{t}\right)^{T} P_{1} G x_{t} \\
V_{2}\left(x_{t}\right) & =\int_{t-h}^{t} x^{T}(\alpha) Q_{1} x(\alpha) d \alpha+\int_{t-\tau(t)}^{t} x^{T}(\theta) S_{1} x(\theta) d \theta, \\
V_{3}\left(x_{t}\right) & =\int_{t-2 h}^{t-h} x^{T}(\alpha) Q_{2} x(\alpha) d \alpha+\int_{t-\tau(t)-h}^{t} x^{T}(\theta) S_{2} x(\theta) d \theta, \\
V_{4}\left(x_{t}\right) & =\int_{t-h}^{t} \dot{x}^{T}(\alpha) Q_{3} \dot{x}(\alpha) d \alpha, \\
V_{5}\left(x_{t}\right) & =\int_{-h}^{0} \int_{t+s}^{t} \xi^{T}(\alpha) Z_{1} \xi(\alpha) d \alpha d s+\int_{-\tau}^{0} \int_{t+s}^{t} \xi^{T}(\theta) R_{1} \xi(\theta) d \theta d s, \\
V_{6}\left(x_{t}\right) & =\int_{-h}^{0} \int_{t+s}^{t} \dot{x}^{T}(\alpha) Z_{2} \dot{x}(\alpha) d \alpha d s+\int_{-\tau}^{0} \int_{t+s}^{t} \dot{x}^{T}(\theta) R_{2} \dot{x}(\theta) d \theta d s,
\end{aligned}
$$

where $x_{t}(\theta)=x(t+\theta),-2 H \leq \theta \leq 0$, and the matrices involved satisfy $P>0, P_{1}>0, Q_{1}>0$, $Q_{2}>0, Q_{3}>0, R_{i}>0, S_{i}>0$, and $Z_{i}>0, i=1,2$. Now calculate the derivative of $V\left(x_{t}\right)$ along the trajectory of the system given in Equation (5):

$$
\begin{aligned}
\dot{V}_{1}\left(x_{t}\right)= & 2 x^{T}(t) P \dot{x}(t)+2\left(G x_{t}\right)^{T} P_{1} \dot{G} x_{t} \\
= & 2 x^{T}(t)\left(P A+P_{1} A\right) x(t)+2 x^{T}(t)\left(P A_{1}+P_{1} A_{1}\right) x(t-\tau(t))-2 x^{T}(t) A^{T} P_{1} C x(t-h) \\
& \quad+2 x^{T}(t) P C \dot{x}(t-h)-2 x^{T}(t-\tau(t)) A_{1}^{T} P_{1} C x(t-h) . \\
\dot{V}_{2}\left(x_{t}\right)= & x^{T}(t) Q_{1} x(t)-x^{T}(t-h) Q_{1} x(t-h)+x^{T}(t) S_{1} x(t) \\
& \quad-(1-\dot{\tau}(t)) x^{T}(t-\tau(t)) S_{1} x(t-\tau(t)) \\
\leq & x^{T}(t)\left(Q_{1}+S_{1}\right) x(t)-x^{T}(t-h) Q_{1} x(t-h)-(1-\mu) x^{T}(t-\tau(t)) S_{1} x(t-\tau(t)) . \quad(17) \\
\dot{V}_{3}\left(x_{t}\right)= & x^{T}(t-h) Q_{2} x(t-h)-x^{T}(t-2 h) Q_{2} x(t-2 h)+x^{T}(t) S_{2} x(t) \\
& \quad-(1-\dot{\tau}(t)) x^{T}(t-\tau(t)-h) S_{2} x(t-\tau(t)-h) \\
\leq & x^{T}(t-h) Q_{2} x(t-h)-x^{T}(t-2 h) Q_{2} x(t-2 h)+x^{T}(t) S_{2} x(t) \\
& \quad-(1-\mu) x^{T}(t-\tau(t)-h) S_{2} x(t-\tau(t)-h) . \\
\dot{V}_{4}\left(x_{t}\right)= & \dot{x}^{T}(t) Q_{3} \dot{x}(t)-\dot{x}^{T}(t-h) Q_{3} \dot{x}(t-h) . \\
\dot{V}_{5}\left(x_{t}\right)= & h \xi^{T}(t) Z_{1} \xi(t)-\int_{t-h}^{t} \xi^{T}(\alpha) Z_{1} \xi(\alpha) d \alpha+\tau \xi^{T}(t) R_{1} \xi(t)-\int_{t-\tau}^{t} \xi^{T}(\theta) R_{1} \xi(\theta) d \theta \\
\leq & \xi^{T}(t)\left[h Z_{1}+\tau R_{1}\right] \xi(t)-\int_{t-h}^{t} \xi^{T}(\alpha) Z_{1} \xi(\alpha) d \alpha-\int_{t-\tau(t)}^{t} \xi^{T}(\theta) R_{1} \xi(\theta) d \theta . \\
\dot{V}_{6}\left(x_{t}\right)= & h \dot{x}^{T}(t) Z_{2} \dot{x}(t)-\int_{t-h}^{t} \dot{x}^{T}(\alpha) Z_{2} \dot{x}(\alpha) d \alpha+\tau \dot{x}^{T}(t) R_{2} \dot{x}(t)-\int_{t-\tau}^{t} \dot{x}^{T}(\theta) R_{2} \dot{x}(\theta) d \theta
\end{aligned}
$$




$$
\leq \dot{x}^{T}(t)\left[h Z_{2}+\tau R_{2}\right] \dot{x}(t)-\int_{t-h}^{t} \dot{x}^{T}(\alpha) Z_{2} \dot{x}(\alpha) d \alpha-\int_{t-\tau(t)}^{t} \dot{x}^{T}(\theta) R_{2} \dot{x}(\theta) d \theta .
$$

Let $S=Q_{3}+h Z_{2}+\tau R_{2}$, and $M=h Z_{1}+\tau R_{1}$. Since

$$
\begin{aligned}
& \dot{x}^{T}(t)\left[Q_{3}+h Z_{2}+\tau R_{2}\right] \dot{x}(t) \\
=\quad & \left.A x(t)+A_{1} x(t-\tau(t))+C \dot{x}(t-h)\right]^{T} S\left[A x(t)+A_{1} x(t-\tau(t))+C \dot{x}(t-h)\right] \\
=\quad & x^{T}(t) A^{T} S A x(t)+2 x^{T}(t) A^{T} S A_{1} x(t-\tau(t)) \\
& \quad+2 x^{T}(t) A^{T} S C \dot{x}(t-h)+x^{T}(t-\tau(t)) A_{1}^{T} S A_{1} x(t-\tau(t)) \\
& \quad+2 x^{T}(t-\tau(t)) A_{1}^{T} S C \dot{x}(t-h)+\dot{x}^{T}(t-h) C^{T} S C \dot{x}(t-h),
\end{aligned}
$$

and

$$
\begin{aligned}
\xi^{T}(t)\left[h Z_{1}+\tau R_{1}\right] \xi(t)= & {\left[A x(t)+A_{1} x(t-\tau(t))\right]^{T} M\left[A x(t)+A_{1} x(t-\tau(t))\right] } \\
= & x^{T}(t) A^{T} M A x(t)+2 x^{T}(t) A^{T} M A_{1} x(t-\tau(t)) \\
& +x^{T}(t-\tau(t)) A_{1}^{T} M A_{1} x(t-\tau(t)),
\end{aligned}
$$

adding Equations (16)-(21) yields

$$
\begin{aligned}
\dot{V}\left(x_{t}\right)= & \dot{V}_{1}\left(x_{t}\right)+\dot{V}_{2}\left(x_{t}\right)+\dot{V}_{3}\left(x_{t}\right)+\dot{V}_{4}\left(x_{t}\right)+\dot{V}_{5}\left(x_{t}\right)+\dot{V}_{6}\left(x_{t}\right) \\
\leq \quad x^{T}(t)\left[2 P A+2 P_{1} A+Q_{1}+S_{1}+S_{2}+A^{T}(S+M) A\right] x(t) & \\
& +2 x^{T}(t)\left[P A_{1}+P_{1} A_{1}+A^{T}(S+M) A_{1}\right] x(t-\tau(t)) \\
& -2 x^{T}(t) A^{T} P_{1} C x(t-h)+2 x^{T}(t)\left[P C+A^{T} S C\right] \dot{x}(t-h) \\
& +x^{T}(t-\tau(t))\left[-(1-\mu) S_{1}+A_{1}^{T}(S+M) A_{1}\right] x(t-\tau(t)) \\
& -2 x^{T}(t-\tau(t)) A_{1}^{T} P_{1} C x(t-h)+2 x^{T}(t-\tau(t)) A_{1}^{T} S C \dot{x}(t-h) \\
& -(1-\mu) x^{T}(t-\tau(t)-h) S_{2} x(t-\tau(t)-h)+x^{T}(t-h)\left[-Q_{1}+Q_{2}\right] x(t-h) \\
& +x^{T}(t-2 h)\left[-Q_{2}\right] x(t-2 h)+\dot{x}^{T}(t-h)\left[-Q_{3}+C^{T} S C\right] \dot{x}(t-h) \\
& -\int_{t-h}^{t} \xi^{T}(\alpha) Z_{1} \xi(\alpha) d \alpha-\int_{t-h}^{t} \dot{x}^{T}(\alpha) Z_{2} \dot{x}(\alpha) d \alpha \\
& -\int_{t-\tau(t)}^{t} \xi^{T}(\theta) R_{1} \xi(\theta) d \theta-\int_{t-\tau(t)}^{t} \dot{x}^{T}(\theta) R_{2} \dot{x}(\theta) d \theta .
\end{aligned}
$$

Since $\dot{x}(t)-C \dot{x}(t-h)=A x(t)+A_{1} x(t-\tau(t))$ and $\xi(t)=A x(t)+A_{1} x(t-\tau(t))$, then

$$
x(t)-C x(t-h)-x(t-\tau(t))+C x(t-\tau(t)-h)=\int_{t-\tau(t)}^{t} \xi(\theta) d \theta
$$

and

$$
x(t)-(C+I) x(t-h)+C x(t-2 h)=\int_{t-h}^{t} \xi(\alpha) d \alpha .
$$

From the Leibniz-Newton Formula, we have that

$$
\begin{aligned}
x(t)-x(t-\tau(t)) & =\int_{t-\tau(t)}^{t} \dot{x}(\theta) d \theta, \\
x(t)-x(t-h) & =\int_{t-h}^{t} \dot{x}(\alpha) d \alpha .
\end{aligned}
$$

Therefore, for any matrices $T_{i}, i=1, \ldots, 6$ of appropriate dimension

$$
2\left[x^{T}(t) T_{1}+x^{T}(t-\tau(t)) T_{2}+x^{T}(t-\tau(t)-h) T_{3}+x^{T}(t-h) T_{4}+x^{T}(t-2 h) T_{5}\right.
$$




$$
\begin{aligned}
& \left.+\dot{x}^{T}(t-h) T_{6}\right][x(t)-C x(t-h)-x(t-\tau(t))+C x(t-\tau(t)-h)] \\
& -2\left[x^{T}(t) T_{1}+x^{T}(t-\tau(t)) T_{2}+x^{T}(t-\tau(t)-h) T_{3}+x^{T}(t-h) T_{4}\right. \\
& \left.+x^{T}(t-2 h) T_{5}+\dot{x}^{T}(t-h) T_{6}\right] \int_{t-\tau(t)}^{t} \xi(\theta) d \theta=0,
\end{aligned}
$$

and so

$$
\begin{gathered}
X^{T}\left[\begin{array}{cccccc}
T_{1}+T_{1}^{T} & -T_{1}+T_{2}^{T} & T_{1} C+T_{3}^{T} & -T_{1} C+T_{4}^{T} & T_{5}^{T} & T_{6}^{T} \\
* & -T_{2}-T_{2}^{T} & T_{2} C-T_{3}^{T} & -T_{2} C-T_{4}^{T} & -T_{5}^{T} & -T_{6}^{T} \\
* & * & T_{3} C+C^{T} T_{3}^{T} & -T_{3} C+C^{T} T_{4}^{T} & C^{T} T_{5}^{T} & C^{T} T_{6}^{T} \\
* & * & * & -T_{4} C-C^{T} T_{4}^{T} & -C^{T} T_{5}^{T} & -C^{T} T_{6}^{T} \\
* & * & * & * & 0 & 0 \\
* & * & * & * & * & 0
\end{array}\right] X \\
-2 \int_{t-\tau(t)}^{t} X^{T}\left[\begin{array}{c}
T_{1} \\
T_{2} \\
T_{3} \\
T_{4} \\
T_{5} \\
T_{6}
\end{array}\right] \xi(\theta) d \theta=0,
\end{gathered}
$$

where

$$
X=\left[\begin{array}{l}
x(t) \\
x(t-\tau(t)) \\
x(t-\tau(t)-h) \\
x(t-h) \\
x(t-2 h) \\
\dot{x}(t-h)
\end{array}\right] .
$$

Similarly, there exist matrices $Y_{i}, N_{i}$, and $W_{i},(i=1, \ldots, 6)$ associated with Equations (26)-(28), respectively, such that

$$
\begin{aligned}
& X^{T}\left[\begin{array}{cccccc}
2 Y_{1} & Y_{2}^{T} & Y_{3}^{T} & -Y_{1}(C+I)+Y_{4}^{T} & Y_{1} C+Y_{5}^{T} & Y_{6}^{T} \\
* & 0 & 0 & -Y_{2}(C+I) & Y_{2} C & 0 \\
* & * & 0 & -Y_{3}(C+I) & Y_{3} C & 0 \\
* & * & * & -2 Y_{4}(C+I) & Y_{4} C-(C+I)^{T} Y_{5}^{T} & -(C+I)^{T} Y_{6}^{T} \\
* & * & * & * & Y_{5} C+C^{T} Y_{5}^{T} & C^{T} Y_{6}^{T} \\
* & * & * & * & * & 0
\end{array}\right] X \\
& -2 \int_{t-h}^{t} X^{T}\left[\begin{array}{c}
Y_{1} \\
Y_{2} \\
Y_{3} \\
Y_{4} \\
Y_{5} \\
Y_{6}
\end{array}\right] \xi(\alpha) d \alpha=0 \\
& X^{T}\left[\begin{array}{cccccc}
N_{1}+N_{1}^{T} & -N_{1}+N_{2}^{T} & N_{3}^{T} & N_{4}^{T} & N_{5}^{T} & N_{6}^{T} \\
* & -N_{2}-N_{2}^{T} & -N_{3}^{T} & -N_{4}^{T} & -N_{5}^{T} & -N_{6}^{T} \\
* & * & 0 & 0 & 0 & 0 \\
* & * & * & 0 & 0 & 0 \\
* & * & * & * & 0 & 0 \\
* & * & * & * & * & 0
\end{array}\right] X \\
& -2 \int_{t-\tau(t)}^{t} X^{T}\left[\begin{array}{c}
N_{1} \\
N_{2} \\
N_{3} \\
N_{4} \\
N_{5} \\
N_{6}
\end{array}\right] \dot{x}(\theta) d \theta=0
\end{aligned}
$$




$$
\begin{gathered}
X^{T}\left[\begin{array}{cccccc}
W_{1}+W_{1}^{T} & W_{2}^{T} & W_{3}^{T} & -W_{1}+W_{4}^{T} & W_{5}^{T} & W_{6}^{T} \\
* & 0 & 0 & -W_{2} & 0 & 0 \\
* & * & 0 & -W_{3} & 0 & 0 \\
* & * & * & -W_{4}-W_{4}^{T} & -W_{5}^{T} & -W_{6}^{T} \\
* & * & * & * & 0 & 0 \\
* & * & * & * & * & 0
\end{array}\right] X \\
-2 \int_{t-h}^{t} X^{T}\left[\begin{array}{c}
W_{1} \\
W_{2} \\
W_{3} \\
W_{4} \\
W_{5} \\
W_{6}
\end{array}\right] \dot{x}(\alpha) d \alpha=0 .
\end{gathered}
$$

Combining Equations (30)-(33) with Inequality (24) and noting that $\tau(t) \leq \tau$, we have

$$
\begin{aligned}
& \dot{V}\left(x_{t}\right) \leq x^{T}(t)\left[P A+A^{T} P+P_{1} A+A^{T} P_{1}+Q_{1}+S_{1}+S_{2}+A^{T}(S+M) A\right. \\
& \left.+Y_{1}+Y_{1}^{T}+T_{1}+T_{1}^{T}+W_{1}+W_{1}^{T}+N_{1}+N_{1}^{T}\right] x(t) \\
& +2 x^{T}(t)\left[P A_{1}+P_{1} A_{1}+A^{T}(S+M) A_{1}+Y_{2}^{T}-T_{1}+T_{2}^{T}+W_{2}^{T}-N_{1}+N_{2}^{T}\right] x(t-\tau(t)) \\
& +2 x^{T}(t)\left[Y_{3}^{T}+T_{1} C+T_{3}^{T}+W_{3}^{T}+N_{3}^{T}\right] x(t-\tau(t)-h) \\
& +2 x^{T}(t)\left[-A^{T} P_{1} C-Y_{1}(C+I)+Y_{4}^{T}-T_{1} C+T_{4}^{T}-W_{1}+W_{4}^{T}+N_{4}^{T}\right] x(t-h) \\
& +2 x^{T}(t)\left[Y_{1} C+Y_{5}^{T}+T_{5}^{T}+W_{5}^{T}+N_{5}^{T}\right] x(t-2 h) \\
& +2 x^{T}(t)\left[P C+A^{T} S C+Y_{6}^{T}+T_{6}^{T}+W_{6}^{T}+N_{6}^{T}\right] \dot{x}(t-h) \\
& +x^{T}(t-\tau(t))\left[-(1-\mu) S_{1}+A_{1}^{T}(S+M) A_{1}-T_{2}-T_{2}^{T}-N_{2}-N_{2}^{T}\right] x(t-\tau(t)) \\
& +2 x^{T}(t-\tau(t))\left[T_{2} C-T_{3}^{T}-N_{3}^{T}\right] x(t-\tau(t)-h) \\
& +2 x(t-\tau(t))\left[-A_{1}^{T} P_{1} C-Y_{2}(C+I)-T_{2} C-T_{4}^{T}-W_{2}-N_{4}^{T}\right] x(t-h) \\
& +2 x(t-\tau(t))\left[Y_{2} C-T_{5}^{T}-N_{5}^{T}\right] x(t-2 h) \\
& +2 x^{T}(t-\tau(t))\left[A_{1}^{T} S C-T_{6}^{T}-N_{6}^{T}\right] \dot{x}(t-h) \\
& +x^{T}(t-\tau(t)-h)\left[-(1-\mu) S_{2}+T_{3} C+C^{T} T_{3}^{T}\right] x(t-\tau(t)-h) \\
& +2 x^{T}(t-\tau(t)-h)\left[-Y_{3}(C+I)-T_{3} C+C^{T} T_{4}^{T}-W_{3}\right] x(t-h) \\
& +2 x^{T}(t-\tau(t)-h)\left[Y_{3} C+C^{T} T_{5}^{T}\right] x(t-2 h) \\
& +2 x^{T}(t-\tau(t)-h)\left[C^{T} T_{6}^{T}\right] \dot{x}(t-h) \\
& +x^{T}(t-h)\left[-Q_{1}+Q_{2}-Y_{4}(C+I)-(C+I)^{T} Y_{4}^{T}-T_{4} C-C^{T} T_{4}^{T}-W_{4}-W_{4}^{T}\right] x(t-h) \\
& +2 x^{T}(t-h)\left[Y_{4} C-(C+I)^{T} Y_{5}^{T}-C^{T} T_{5}^{T}-W_{5}^{T}\right] x(t-2 h) \\
& +2 x^{T}(t-h)\left[-(C+I)^{T} Y_{6}^{T}-C^{T} T_{6}^{T}-W_{6}^{T}\right] \dot{x}(t-h) \\
& +x^{T}(t-2 h)\left[-Q_{2}+Y_{5} C+C^{T} Y_{5}^{T}\right] x(t-2 h) \\
& +2 x^{T}(t-2 h)\left[C^{T} Y_{6}^{T}\right] \dot{x}(t-h)+\dot{x}^{T}(t-h)\left[-Q_{3}+C^{T} S C\right] \dot{x}(t-h) \\
& -\frac{1}{h} \int_{t-h}^{t} h \xi^{T}(\alpha) \frac{1}{h} Z_{1} h \xi(\alpha) d \alpha-\frac{1}{h} \int_{t-h}^{t} h \dot{x}^{T}(\alpha) \frac{1}{h} Z_{2} h \dot{x}(\alpha) d \alpha \\
& -\frac{1}{\tau(t)} \int_{t-\tau(t)}^{t} \tau(t) \xi^{T}(\theta) \frac{1}{\tau} R_{1} \tau(t) \xi(\theta) d \theta-\frac{1}{\tau(t)} \int_{t-\tau(t)}^{t} \tau(t) \dot{x}^{T}(\theta) \frac{1}{\tau} R_{2} \tau(t) \dot{x}(\theta) d \theta \\
& -\frac{2}{h} \int_{t-h}^{t} X^{T}\left[\begin{array}{c}
Y_{1} \\
Y_{2} \\
Y_{3} \\
Y_{4} \\
Y_{5} \\
Y_{6}
\end{array}\right] h \xi(\alpha) d \alpha-\frac{2}{\tau(t)} \int_{t-\tau(t)}^{t} X^{T}\left[\begin{array}{c}
T_{1} \\
T_{2} \\
T_{3} \\
T_{4} \\
T_{5} \\
T_{6}
\end{array}\right] \tau(t) \xi(\theta) d \theta-
\end{aligned}
$$




$$
\begin{aligned}
& \frac{2}{h} \int_{t-h}^{t} X^{T}\left[\begin{array}{c}
W_{1} \\
W_{2} \\
W_{3} \\
W_{4} \\
W_{5} \\
W_{6}
\end{array}\right] h \dot{x}(\alpha) d \alpha-\frac{2}{\tau(t)} \int_{t-\tau(t)}^{t} X^{T}\left[\begin{array}{c}
N_{1} \\
N_{2} \\
N_{3} \\
N_{4} \\
N_{5} \\
N_{6}
\end{array}\right] \tau(t) \dot{x}(\theta) d \theta \\
& =\frac{1}{h \tau(t)} \int_{t-h}^{t} d \alpha \int_{t-\tau(t)}^{t} \eta^{T}(t, h, \theta, \alpha) \Omega \eta(t, h, \theta, \alpha) d \theta, \\
& \text { where } \eta(t, h, \theta, \alpha)=\left[\begin{array}{c}
x(t) \\
x(t-\tau(t)) \\
x(t-\tau(t)-h) \\
x(t-h) \\
x(t-2 h) \\
\dot{x}(t-h) \\
\tau(t) \xi(\theta) \\
\tau(t) \dot{x}(\theta) \\
h \xi(\alpha) \\
h \dot{x}(\alpha)
\end{array}\right] \\
& \Omega=\left[\begin{array}{cccccccccc}
\Omega_{11} & \Omega_{12} & \Omega_{13} & \Omega_{14} & \Omega_{15} & \Omega_{16} & -T_{1} & -N_{1} & -Y_{1} & -W_{1} \\
* & \Omega_{22} & \Omega_{23} & \Omega_{24} & \Omega_{25} & \Omega_{26} & -T_{2} & -N_{2} & -Y_{2} & -W_{2} \\
* & * & \Omega_{33} & \Omega_{34} & \Omega_{35} & \Omega_{36} & -T_{3} & -N_{3} & -Y_{3} & -W_{3} \\
* & * & * & \Omega_{44} & \Omega_{45} & \Omega_{46} & -T_{4} & -N_{4} & -Y_{4} & -W_{4} \\
* & * & * & * & \Omega_{55} & \Omega_{56} & -T_{5} & -N_{5} & -Y_{5} & -W_{5} \\
* & * & * & * & * & \Omega_{66} & -T_{6} & -N_{6} & -Y_{6} & -W_{6} \\
* & * & * & * & * & * & -\frac{R_{1}}{\tau} & 0 & 0 & 0 \\
* & * & * & * & * & * & * & -\frac{R_{2}}{\tau} & 0 & 0 \\
* & * & * & * & * & * & * & * & -\frac{Z_{1}}{h} & 0 \\
* & * & * & * & * & * & * & * & * & -\frac{Z_{2}}{h}
\end{array}\right],
\end{aligned}
$$

and

$$
\begin{aligned}
\Omega_{11}= & P A+A^{T} P+P_{1} A+A^{T} P_{1}+Q_{1}+S_{1}+S_{2}+A^{T}(S+M) A+ \\
& Y_{1}+Y_{1}^{T}+T_{1}+T_{1}^{T}+W_{1}+W_{1}^{T}+N_{1}+N_{1}^{T}, \\
\Omega_{12}= & P A_{1}+P_{1} A_{1}+A^{T}(S+M) A_{1}+Y_{2}^{T}-T_{1}+T_{2}^{T}+W_{2}^{T}-N_{1}+N_{2}^{T}, \\
\Omega_{13}= & Y_{3}^{T}+T_{1} C+T_{3}^{T}+W_{3}^{T}+N_{3}^{T}, \\
\Omega_{14}= & -A^{T} P_{1} C-Y_{1}(C+I)+Y_{4}^{T}-T_{1} C+T_{4}^{T}-W_{1}+W_{4}^{T}+N_{4}^{T}, \\
\Omega_{15}= & Y_{1} C+Y_{5}^{T}+T_{5}^{T}+W_{5}^{T}+N_{5}^{T}, \\
\Omega_{16}= & P C+A^{T} S C+Y_{6}^{T}+T_{6}^{T}+W_{6}^{T}+N_{6}^{T}, \\
\Omega_{22}= & -(1-\mu) S_{1}+A_{1}^{T}(S+M) A_{1}-T_{2}-T_{2}^{T}-N_{2}-N_{2}^{T}, \\
\Omega_{23}= & T_{2} C-T_{3}^{T}-N_{3}^{T}, \\
\Omega_{24}= & -A_{1}^{T} P_{1} C-Y_{2}(C+I)-T_{2} C-T_{4}^{T}-W_{2}-N_{4}^{T}, \\
\Omega_{25}= & Y_{2} C-T_{5}^{T}-N_{5}^{T}, \\
\Omega_{26}= & A_{1}^{T} S C-T_{6}^{T}-N_{6}^{T}, \\
\Omega_{33}= & -(1-\mu) S_{2}+T_{3} C+C^{T} T_{3}^{T}, \\
\Omega_{34}= & -Y_{3}(C+I)-T_{3} C+C^{T} T_{4}^{T}-W_{3}, \\
\Omega_{35}= & Y_{3} C+C^{T} T_{5}^{T}, \\
\Omega_{36}= & C^{T} T_{6}^{T}, \\
\Omega_{44}= & -Q_{1}+Q_{2}-Y_{4}(C+I)-(C+I)^{T} Y_{4}^{T}-T_{4} C-C^{T} T_{4}^{T}-W_{4}-W_{4}^{T},
\end{aligned}
$$




$$
\begin{aligned}
\Omega_{45} & =Y_{4} C-(C+I)^{T} Y_{5}^{T}-C^{T} T_{5}^{T}-W_{5}^{T}, \\
\Omega_{46} & =-(C+I)^{T} Y_{6}^{T}-C^{T} T_{6}^{T}-W_{6}^{T}, \\
\Omega_{55} & =-Q_{2}+Y_{5} C+C^{T} Y_{5}^{T}, \\
\Omega_{56} & =C^{T} Y_{6}^{T} \\
\Omega_{66} & =-Q_{3}+C^{T} S C, \\
M & =h Z_{1}+\tau R_{1}, \\
S & =Q_{3}+h Z_{2}+\tau R_{2} .
\end{aligned}
$$

Obviously, $\dot{V}\left(x_{t}\right)<0$ if $\Omega<0$ and $\eta(t, h, \theta, \alpha) \neq 0$. Therefore, when $\Omega<0$, the system given in Equation (5) is asymptotically stable. Applying Schur complement, $\Omega<0$ is equivalent to

$$
\left[\begin{array}{cccccccccccc}
\tilde{\Omega}_{11} & \tilde{\Omega}_{12} & \tilde{\Omega}_{13} & \tilde{\Omega}_{14} & \tilde{\Omega}_{15} & \tilde{\Omega}_{16} & -T_{1} & -N_{1} & -Y_{1} & -W_{1} & A^{T} M & A^{T} S \\
* & \tilde{\Omega}_{22} & \tilde{\Omega}_{23} & \tilde{\Omega}_{24} & \tilde{\Omega}_{25} & \tilde{\Omega}_{26} & -T_{2} & -N_{2} & -Y_{2} & -W_{2} & A_{1}^{T} M & A_{1}^{T} S \\
* & * & \tilde{\Omega}_{33} & \tilde{\Omega}_{34} & \tilde{\Omega}_{35} & \tilde{\Omega}_{36} & -T_{3} & -N_{3} & -Y_{3} & -W_{3} & 0 & 0 \\
* & * & * & \tilde{\Omega}_{44} & \tilde{\Omega}_{45} & \tilde{\Omega}_{46} & -T_{4} & -N_{4} & -Y_{4} & -W_{4} & 0 & 0 \\
* & * & * & * & \tilde{\Omega}_{55} & \tilde{\Omega}_{56} & -T_{5} & -N_{5} & -Y_{5} & -W_{5} & 0 & 0 \\
* & * & * & * & * & \tilde{\Omega}_{66} & -T_{6} & -N_{6} & -Y_{6} & -W_{6} & 0 & C^{T} S \\
* & * & * & * & * & * & -\frac{R_{1}}{\tau} & 0 & 0 & 0 & 0 & 0 \\
* & * & * & * & * & * & * & -\frac{R_{2}}{\tau} & 0 & 0 & 0 & 0 \\
* & * & * & * & * & * & * & * & -\frac{Z_{1}}{h} & 0 & 0 & 0 \\
* & * & * & * & * & * & * & * & * & -\frac{Z_{2}}{h} & 0 & 0 \\
* & * & * & * & * & * & * & * & * & * & -M & 0 \\
* & * & * & * & * & * & * & * & * & * & * & -S
\end{array}\right]<0 .
$$

Multiplying both sides of Inequality (37) by the matrix $\operatorname{diag}(I, I, I, I, I, I, \tau I, \tau I, h I, h I, I, I)$ gives Inequality (8), i.e. $\tilde{\Omega}<0$. This completes the proof of Theorem 1 .

He et al [9] studied the delay-dependent stability of the following neutral system with fixed time delays:

$$
\dot{x}(t)-C \dot{x}(t-h)=A x(t)+A_{1} x(t-\tau),
$$

and obtained a delay-dependent stability criterion. We now restate their result as Corollary 1 , and show that He et al's result in [9] is a special case of our Theorem 1.

Corollary 1. Given scalars $h>0$ and $\tau>0$, the nominal system given in Equation (38) is asymptotically stable if the operator $G$ is stable and there exist positive definite matrices $P_{1}>0$, $S_{1}>0, Q_{1}>0, Q_{3}>0$, non-negative definite matrices $X_{i i} \geq 0, Y_{i i} \geq 0, i=1, \ldots, 5$ and otherwise arbitrary matrices $X_{i j}, Y_{i j}, 1 \leq i<j \leq 5$ such that the following LMIs are satisfied:

$$
\begin{aligned}
\Phi= & {\left[\begin{array}{ccccc}
\Phi_{11} & \Phi_{12} & \Phi_{13} & \Phi_{14} & A^{T} S \\
\Phi_{12}^{T} & \Phi_{22} & \Phi_{23} & \Phi_{24} & A_{1}^{T} S \\
\Phi_{13}^{T} & \Phi_{23}^{T} & \Phi_{33} & \Phi_{34} & 0 \\
\Phi_{14}^{T} & \Phi_{24}^{T} & \Phi_{34}^{T} & \Phi_{44} & C^{T} S \\
S A & S A_{1} & 0 & S C & -S
\end{array}\right]<0, } \\
\Psi= & {\left[\begin{array}{ccccc}
X_{11} & X_{12} & X_{13} & X_{14} & X_{15} \\
X_{12}^{T} & X_{22} & X_{23} & X_{24} & X_{25} \\
X_{13}^{T} & X_{23}^{T} & X_{33} & X_{34} & X_{35} \\
X_{14}^{T} & X_{24}^{T} & X_{34}^{T} & X_{44} & X_{45} \\
X_{15}^{T} & X_{25}^{T} & X_{35}^{T} & X_{45}^{T} & X_{55}
\end{array}\right] \geq 0, } \\
\Xi= & {\left[\begin{array}{cccccc}
Y_{11} & Y_{12} & Y_{13} & Y_{14} & Y_{15} \\
Y_{12}^{T} & Y_{22} & Y_{23} & Y_{24} & Y_{25} \\
Y_{13}^{T} & Y_{23}^{T} & Y_{33} & Y_{34} & Y_{35} \\
Y_{14}^{T} & Y_{24}^{T} & Y_{34}^{T} & Y_{44} & Y_{45} \\
Y_{15}^{T} & Y_{25}^{T} & Y_{35}^{T} & Y_{45}^{T} & Y_{55}
\end{array}\right] \geq 0, }
\end{aligned}
$$


where

$$
\begin{aligned}
\Phi_{11} & =P_{1} A+A^{T} P_{1}+S_{1}+Q_{1}+X_{15}+X_{15}^{T}+Y_{15}+Y_{15}^{T}+\tau X_{11}+h Y_{11} \\
\Phi_{12} & =P_{1} A_{1}-X_{15}+X_{25}^{T}+\tau X_{12}+h Y_{12} \\
\Phi_{13} & =-A^{T} P_{1} C+X_{35}^{T}+Y_{35}^{T}-Y_{15}+\tau X_{13}+h Y_{13} \\
\Phi_{14} & =X_{45}^{T}+Y_{45}^{T}+\tau X_{14}+h Y_{14} \\
\Phi_{22} & =-S_{1}-X_{25}-X_{25}^{T}+\tau X_{22}+h Y_{22} \\
\Phi_{23} & =-A_{1}^{T} P_{1} C-X_{35}-Y_{25}+\tau X_{23}+h Y_{23} \\
\Phi_{24} & =-X_{45}^{T}+\tau X_{24}+h Y_{24} \\
\Phi_{33} & =-Q_{1}-Y_{35}-Y_{35}^{T}+\tau X_{33}+h Y_{33} \\
\Phi_{34} & =-Y_{45}^{T}+\tau X_{34}+h Y_{34} \\
\Phi_{44} & =-Q_{3}+\tau X_{44}+h Y_{44} \\
S & =Q_{3}+\tau X_{55}+h Y_{55}
\end{aligned}
$$

Proof. Case 1: suppose $X_{55}>0, Y_{55}>0$.

Since $\Psi \geq 0$, it is clear that

$$
\left[\begin{array}{cccccc}
X_{11} & X_{12} & X_{13} & X_{14} & 0 & X_{15} \\
X_{12}^{T} & X_{22} & X_{23} & X_{24} & 0 & X_{25} \\
X_{13}^{T} & X_{23}^{T} & X_{33} & X_{34} & 0 & X_{35} \\
X_{14}^{T} & X_{24}^{T} & X_{34}^{T} & X_{44} & 0 & X_{45} \\
0 & 0 & 0 & 0 & 0 & 0 \\
X_{15}^{T} & X_{25}^{T} & X_{35}^{T} & X_{45}^{T} & 0 & X_{55}
\end{array}\right] \geq 0
$$

By Schur Complement,

$$
\left[\begin{array}{ccccc}
X_{11} & X_{12} & X_{13} & X_{14} & 0 \\
X_{12}^{T} & X_{22} & X_{23} & X_{24} & 0 \\
X_{13}^{T} & X_{23}^{T} & X_{33} & X_{34} & 0 \\
X_{14}^{T} & X_{24}^{T} & X_{34}^{T} & X_{44} & 0 \\
0 & 0 & 0 & 0 & 0
\end{array}\right]-\left[\begin{array}{c}
X_{15} \\
X_{25} \\
X_{35} \\
X_{45} \\
0
\end{array}\right] X_{55}^{-1}\left[\begin{array}{lllll}
X_{15}^{T} & X_{25}^{T} & X_{35}^{T} & X_{45}^{T} & 0
\end{array}\right] \geq 0
$$

so

$$
\begin{gathered}
{\left[\begin{array}{ccccc}
\Phi_{11}-\tau X_{11} & \Phi_{12}-\tau X_{12} & \Phi_{13}-\tau X_{13} & \Phi_{14}-\tau X_{14} & A^{T} S \\
\Phi_{12}^{T}-\tau X_{12}^{T} & \Phi_{22}-\tau X_{22} & \Phi_{23}-\tau X_{23} & \Phi_{24}-\tau X_{24} & A_{1}^{T} S \\
\Phi_{13}^{T}-\tau X_{13}^{T} & \Phi_{23}^{T}-\tau X_{23}^{T} & \Phi_{33}-\tau X_{33} & \Phi_{34}-\tau X_{34} & 0 \\
\Phi_{14}^{T}-\tau X_{14}^{T} & \Phi_{24}^{T}-\tau X_{24}^{T} & \Phi_{34}^{T}-\tau X_{34}^{T} & \Phi_{44}-\tau X_{44} & C^{T} S \\
S A & S A_{1} & 0 & S C & -S
\end{array}\right]} \\
+\tau\left[\begin{array}{c}
-X_{15} \\
-X_{25} \\
-X_{35} \\
-X_{45} \\
0
\end{array}\right] X_{55}^{-1}\left[-X_{15}^{T}-X_{25}^{T}-X_{35}^{T}-X_{45}^{T} 0\right]<0 .
\end{gathered}
$$

Applying Schur Complement again, we find that

$$
\left[\begin{array}{cccccc}
\Phi_{11}-\tau X_{11} & \Phi_{12}-\tau X_{12} & \Phi_{13}-\tau X_{13} & \Phi_{14}-\tau X_{14} & -\tau X_{15} & A^{T} S \\
* & \Phi_{22}-\tau X_{22} & \Phi_{23}-\tau X_{23} & \Phi_{24}-\tau X_{24} & -\tau X_{25} & A_{1}^{T} S \\
* & * & \Phi_{33}-\tau X_{33} & \Phi_{34}-\tau X_{34} & -\tau X_{35} & 0 \\
* & * & * & \Phi_{44}-\tau X_{44} & -\tau X_{45} & C^{T} S \\
* & * & * & * & -\tau X_{55} & 0 \\
* & * & * & * & * & -S
\end{array}\right]<0 .
$$


Similarly, since $\Xi \geq 0$, then

$$
\left[\begin{array}{cccccc}
Y_{11} & Y_{12} & Y_{13} & Y_{14} & 0 & 0 \\
Y_{12}^{T} & Y_{22} & Y_{23} & Y_{24} & 0 & 0 \\
Y_{13}^{T} & Y_{23}^{T} & Y_{33} & Y_{34} & 0 & 0 \\
Y_{14}^{T} & Y_{24}^{T} & Y_{34}^{T} & Y_{44} & 0 & 0 \\
0 & 0 & 0 & 0 & 0 & 0 \\
0 & 0 & 0 & 0 & 0 & 0
\end{array}\right]-\left[\begin{array}{c}
Y_{15} \\
Y_{25} \\
Y_{35} \\
Y_{45} \\
0 \\
0
\end{array}\right] Y_{55}^{-1}\left[\begin{array}{llll}
Y_{15}^{T} & Y_{25}^{T} & Y_{35}^{T} Y_{45}^{T} & 0
\end{array}\right] \geq 0
$$

Therefore,

$$
\begin{aligned}
& {\left[\begin{array}{cccccc}
\Phi_{11}-\tau X_{11} & \Phi_{12}-\tau X_{12} & \Phi_{13}-\tau X_{13} & \Phi_{14}-\tau X_{14} & -\tau X_{15} & A^{T} S \\
* & \Phi_{22}-\tau X_{22} & \Phi_{23}-\tau X_{23} & \Phi_{24}-\tau X_{24} & -\tau X_{25} & A_{1}^{T} S \\
* & * & \Phi_{33}-\tau X_{33} & \Phi_{34}-\tau X_{34} & -\tau X_{35} & 0 \\
* & * & * & \Phi_{44}-\tau X_{44} & -\tau X_{45} & C^{T} S \\
* & * & * & * & -\tau X_{55} & 0 \\
* & * & * & * & * & -S
\end{array}\right]} \\
& -h\left[\begin{array}{cccccc}
Y_{11} & Y_{12} & Y_{13} & Y_{14} & 0 & 0 \\
Y_{12}^{T} & Y_{22} & Y_{23} & Y_{24} & 0 & 0 \\
Y_{13}^{T} & Y_{23}^{T} & Y_{33} & Y_{34} & 0 & 0 \\
Y_{14}^{T} & Y_{24}^{T} & Y_{34}^{T} & Y_{44} & 0 & 0 \\
0 & 0 & 0 & 0 & 0 & 0 \\
0 & 0 & 0 & 0 & 0 & 0
\end{array}\right]+h\left[\begin{array}{c}
-Y_{15} \\
-Y_{25} \\
-Y_{35} \\
-Y_{45} \\
0 \\
0
\end{array}\right] Y_{55}^{-1}\left[\begin{array}{lll}
-Y_{15}^{T}-Y_{25}^{T}-Y_{35}^{T}-Y_{45}^{T} & 0 & 0
\end{array}\right]<0
\end{aligned}
$$

Using Schur Complement gives

$$
\left[\begin{array}{ccccccc}
\bar{\Phi}_{11} & \bar{\Phi}_{12} & \bar{\Phi}_{13} & \bar{\Phi}_{14} & -\tau X_{15} & -h Y_{15} & A^{T} S \\
* & \bar{\Phi}_{22} & \bar{\Phi}_{23} & \bar{\Phi}_{24} & -\tau X_{25} & -h Y_{25} & A_{1}^{T} S \\
* & * & \bar{\Phi}_{33} & \bar{\Phi}_{34} & -\tau X_{35} & -h Y_{35} & 0 \\
* & * & * & \bar{\Phi}_{44} & -\tau X_{45} & -h Y_{45} & C^{T} S \\
* & * & * & * & -\tau X_{55} & 0 & 0 \\
* & * & * & * & * & -h Y_{55} & 0 \\
* & * & * & * & * & * & -S
\end{array}\right]<0
$$

where for simplicity, we have introduced the notation $\bar{\Phi}_{i j}=\Phi_{i j}-\tau X_{i j}-h Y_{i j}$.

There exists a positive definite matrix $M$ with appropriate dimension, such that

$$
\begin{aligned}
& {\left[\begin{array}{ccccccc}
\bar{\Phi}_{11} & \bar{\Phi}_{12} & \bar{\Phi}_{13} & \bar{\Phi}_{14} & -\tau X_{15} & -h Y_{15} & A^{T} S \\
* & \bar{\Phi}_{22} & \bar{\Phi}_{23} & \bar{\Phi}_{24} & -\tau X_{25} & -h Y_{25} & A_{1}^{T} S \\
* & * & \bar{\Phi}_{33} & \bar{\Phi}_{34} & -\tau X_{35} & -h Y_{35} & 0 \\
* & * & * & \bar{\Phi}_{44} & -\tau X_{45} & -h Y_{45} & C^{T} S \\
* & * & * & * & -\tau X_{55} & 0 & 0 \\
* & * & * & * & * & -h Y_{55} & 0 \\
* & * & * & * & * & * & -S
\end{array}\right]} \\
& +\left[\begin{array}{c}
A^{T} M \\
A_{1}^{T} M \\
0 \\
0 \\
0 \\
0 \\
0
\end{array}\right] M^{-1}\left[\begin{array}{llllll}
M A & M A_{1} & 0 & 0 & 0 & 0
\end{array}\right]<0
\end{aligned}
$$


By Schur Complement,

$$
\left[\begin{array}{cccccccc}
\bar{\Phi}_{11} & \bar{\Phi}_{12} & \bar{\Phi}_{13} & \bar{\Phi}_{14} & -\tau X_{15} & -h Y_{15} & A^{T} M & A^{T} S \\
* & \bar{\Phi}_{22} & \bar{\Phi}_{23} & \bar{\Phi}_{24} & -\tau X_{25} & -h Y_{25} & A_{1}^{T} M & A_{1}^{T} S \\
* & * & \bar{\Phi}_{33} & \bar{\Phi}_{34} & -\tau X_{35} & -h Y_{35} & 0 & 0 \\
* & * & * & \bar{\Phi}_{44} & -\tau X_{45} & -h Y_{45} & 0 & C^{T} S \\
* & * & * & * & -\tau X_{55} & 0 & 0 & 0 \\
* & * & * & * & * & -h Y_{55} & 0 & 0 \\
* & * & * & * & * & * & -M & 0 \\
* & * & * & * & * & * & * & -S
\end{array}\right]<0
$$

Since $M$ is a positive definite matrix, it can be decomposed into the sum of two positive definite matrices $\tau R_{1}$ and $h Z_{1}$, i.e. $M=\tau R_{1}+h Z_{1}$. Since $\tau R_{1}$ and $h Z_{1}$ are positive definite, then

$$
\left[\begin{array}{cccccccccc}
\bar{\Phi}_{11} & \bar{\Phi}_{12} & \bar{\Phi}_{13} & \bar{\Phi}_{14} & 0 & -\tau X_{15} & 0 & -h Y_{15} & A^{T} M & A^{T} S \\
* & \bar{\Phi}_{22} & \bar{\Phi}_{23} & \bar{\Phi}_{24} & 0 & -\tau X_{25} & 0 & -h Y_{25} & A_{1}^{T} M & A_{1}^{T} S \\
* & * & \bar{\Phi}_{33} & \bar{\Phi}_{34} & 0 & -\tau X_{35} & 0 & -h Y_{35} & 0 & 0 \\
* & * & * & \bar{\Phi}_{44} & 0 & -\tau X_{45} & 0 & -h Y_{45} & 0 & C^{T} S \\
* & * & * & * & -\tau R_{1} & 0 & 0 & 0 & 0 & 0 \\
* & * & * & * & * & -\tau X_{55} & 0 & 0 & 0 & 0 \\
* & * & * & * & * & * & -h Z_{1} & 0 & 0 & 0 \\
* & * & * & * & * & * & * & -h Y_{55} & 0 & 0 \\
* & * & * & * & * & * & * & * & -M & 0 \\
* & * & * & * & * & * & * & * & * & -S
\end{array}\right]<0 .
$$

We can now choose appropriate positive definite matrices $S_{2}$ and $Q_{2}$ such that

$$
\left[\begin{array}{cccccccccccc}
\bar{\Phi}_{11}+S_{2} & \bar{\Phi}_{12} & 0 & \bar{\Phi}_{13} & 0 & \bar{\Phi}_{14} & 0 & -\tau X_{15} & 0 & -h Y_{15} & A^{T} M & A^{T} S \\
* & \bar{\Phi}_{22} & 0 & \bar{\Phi}_{23} & 0 & \bar{\Phi}_{24} & 0 & -\tau X_{25} & 0 & -h Y_{25} & A_{1}^{T} M & A_{1}^{T} S \\
* & * & -S_{2} & 0 & 0 & 0 & 0 & 0 & 0 & 0 & 0 & 0 \\
* & * & * & \bar{\Phi}_{33}+Q_{2} & 0 & \bar{\Phi}_{34} & 0 & -\tau X_{35} & 0 & -h Y_{35} & 0 & 0 \\
* & * & * & * & -Q_{2} & 0 & 0 & 0 & 0 & 0 & 0 & 0 \\
* & * & * & * & * & \bar{\Phi}_{44} & 0 & -\tau X_{45} & 0 & -h Y_{45} & 0 & C^{T} S \\
* & * & * & * & * & * & -\tau R_{1} & 0 & 0 & 0 & 0 & 0 \\
* & * & * & * & * & * & * & -\tau X_{55} & 0 & 0 & 0 & 0 \\
* & * & * & * & * & * & * & * & -h Z_{1} & 0 & 0 & 0 \\
* & * & * & * & * & * & * & * & * & -h Y_{55} & 0 & 0 \\
* & * & * & * & * & * & * & * & * & * & -M & 0 \\
* & * & * & * & * & * & * & * & * & * & * & -S
\end{array}\right]<0 .
$$

The proof of Theorem 1 makes it clear that if the positive matrix $P$ in Theorem 1 is replaced by a non-negative matrix, Theorem 1 still holds. Let $P=0, R_{2}=X_{55}, Z_{2}=Y_{55}, \mu=0, N_{1}=X_{15}$, $N_{2}=X_{25}, N_{3}=0, N_{4}=X_{35}, N_{5}=0, N_{6}=X_{45}, W_{1}=Y_{15}, W_{2}=Y_{25}, W_{3}=0, W_{4}=Y_{35}$, $W_{5}=0, W_{6}=Y_{45}, T_{i}=Y_{i}=0, i=1, \ldots, 6$. Then Corollary 1 for the case $X_{55}>0, Y_{55}>0$ can be obtained from Theorem 1 .

Case 2: Suppose $X_{55}=0, Y_{55}=0$.

Since $\Phi<0$, there exists some small $\varepsilon>0$ such that

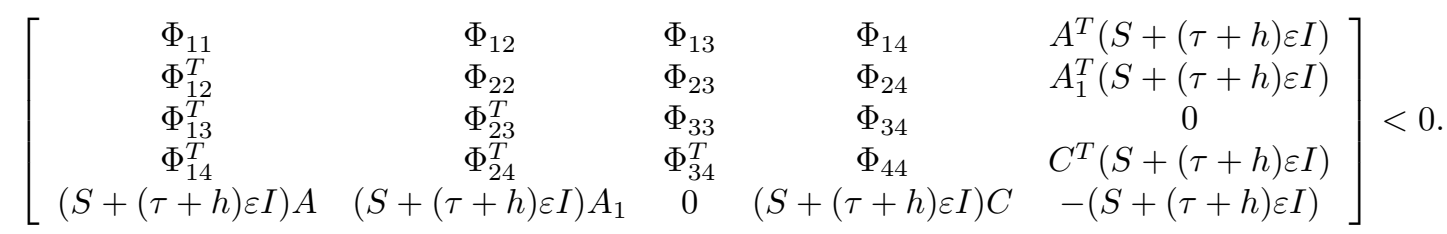

Furthermore, since $\Psi \geq 0$ and $\Xi \geq 0$, it is clear that

$$
\left[\begin{array}{ccccc}
X_{11} & X_{12} & X_{13} & X_{14} & X_{15} \\
X_{12}^{T} & X_{22} & X_{23} & X_{24} & X_{25} \\
X_{13}^{T} & X_{23}^{T} & X_{33} & X_{34} & X_{35} \\
X_{14}^{T} & X_{24}^{T} & X_{34}^{T} & X_{44} & X_{45} \\
X_{15}^{T} & X_{25}^{T} & X_{35}^{T} & X_{45}^{T} & \varepsilon I
\end{array}\right] \geq 0
$$


and

$$
\left[\begin{array}{ccccc}
Y_{11} & Y_{12} & Y_{13} & Y_{14} & Y_{15} \\
Y_{12}^{T} & Y_{22} & Y_{23} & Y_{24} & Y_{25} \\
Y_{13}^{T} & Y_{23}^{T} & Y_{33} & Y_{34} & Y_{35} \\
Y_{14}^{T} & Y_{24}^{T} & Y_{34}^{T} & Y_{44} & Y_{45} \\
Y_{15}^{T} & Y_{25}^{T} & Y_{35}^{T} & Y_{45}^{T} & \varepsilon I
\end{array}\right] \geq 0
$$

Let $\bar{X}_{55}=\bar{Y}_{55}=\varepsilon I$ and $\bar{S}=Q_{3}+\tau \bar{X}_{55}+h \bar{Y}_{55}$. Then Corollary 1 in the case $X_{55}=0, Y_{55}=0$ can be proved by following the same lines of proof as for Case 1 .

Case 3: $X_{55}=0, Y_{55}>0$ or $X_{55}>0, Y_{55}=0$.

Following a similar approach to that used for Case 2, it is easy to show that Corollary 1 holds for $X_{55}=0, Y_{55}>0$, or $X_{55}>0, Y_{55}=0$.

This completes the proof of Corollary 1.

A retarded system is a special case of a neutral system with mixed delays, so we now compare our method and existing methods in the literature both for retarded systems, and more general neutral systems with delays.

Setting $C=0, h=0$ in Equation (5) causes the associated neutral systems with mixed delays to become retarded systems. By slightly modifying the proof of Theorem 1, we can obtain Corollary 2 from Theorem 1 .

Corollary 2. If $0 \leq \tau(t) \leq \tau, \dot{\tau}(t) \leq \mu<1$, then the linear retarded system $\dot{x}(t)=A x(t)+$ $A_{1} x(t-\tau(t))$ is asymptotically stable if there exist matrices $P>0, Q>0, R>0, T_{1}$ and $T_{2}$ such that the following LMI holds:

$$
\left[\begin{array}{cccc}
P A+A^{T} P+T_{1}+T_{1}^{T}+Q & -T_{1}+P A_{1}+T_{2}^{T} & -\tau T_{1} & \tau A^{T} R \\
* & -T_{2}-T_{2}^{T}-(1-\mu) Q & -\tau T_{2} & \tau A_{1}^{T} R \\
* & * & -\tau R & 0 \\
* & * & * & -\tau R
\end{array}\right]<0 .
$$

Remark 1. Xu and Lam [21] studied delay-dependent stability criteria for retarded systems with constant delay - their results are restricted to a time-invariant delay. Wu et al [19] studied the robust stability of retarded systems with time-varying delay and devised delay-dependent stability criteria. Their stability condition includes two complicated linear matrix inequalities (LMI) relating the system matrices of the problem. These LMIs contain many unnecessary free matrix variables which must be stored and computed. In this paper, unlike earlier methods used to derive delay-dependent stability criteria such as those in [3, 4], finding an upper bound on the weighted cross-products of the state and the delayed state is avoided. Thus, inequalities such as those used by Park [18] or Moon [14] to bound these cross terms are not needed. We also introduce various slack matrix variables in the derivative of the Lyapunov functional, which reduces conservatism. Setting $C=0, h=0$ in Equation (1) or Equation (5) causes the associated neutral systems with delays to become retarded systems. Corollary 2 in this paper is a stability criterion for such retarded systems. It is theoretically established that the stability results in this paper are less conservative than those in [3] and [22]. The systems Wu et al [19] studied are limited to linear systems of retarded type with time-varying delay instead of linear neutral systems with mixed time delays, and therefore, the results in [19] are special cases of our results here. Corollary 2 in this paper extends the stability result in [21] to the case of systems with time-varying delay. Corollary 2 is equivalent to Theorem 2 in [19]. Furthermore, Corollary 2 eliminates the unnecessary matrix variables in [19].

Remark 2. A descriptor model transformation was introduced for analysis of delay-dependent stability of neutral systems in [2]. Fridman and Shaked [4] extended the results in [2] to the case of systems with time-varying delays by finding tighter bounds on the cross terms introduced by Park in [18]. This method produces less conservative criteria than those in [11]. However, since the basic approach in [4] is based on the substitution of $x(t)-\int_{t-\tau}^{t} \dot{x}(s) d s$ for $x(t-\tau)$, and Park's inequality for bounding of the cross terms, it can not entirely overcome the conservatism of the methods given by Park [18]. Stability criteria obtained in [4] are neutral-delay-independent. Furthermore, these 
stability criteria do not take into account the information contained in the system equation, i.e., relationships between the state vectors $x(t), x(t-\tau(t)), x(t-\tau(t)-h), x(t-h), x(t-2 h)$ and the derivative of the state vector at $x(t-h)$. Our paper presents a new approach to establishing both neutral-delay-dependent and discrete-delay-dependent stability criteria for time-varying-delay systems which makes use of this information without requiring use of Park's inequality [18] or Moon's inequality [14].

Remark 3. Recently, Han [7] studied the stability of linear neutral systems with mixed time delays and time-varying system matrices, using a novel discretized Lyapunov functional approach. The stability criteria obtained by Han are applicable to linear neutral systems with both small and large discrete delays. These criteria show significant improvements over earlier results, but they are only discrete-delay dependent, and are neutral-delay independent. Furthermore, these criteria cannot be applied to neutral systems with time-varying state delay. In contrast, the stability criterion for time-varying-delay systems established in this paper is both neutral-delay dependent and discretedelay dependent.

In the rest of this section, using Theorem 1, we obtain a new delay-dependent robust stability criterion for the neutral system with time-varying uncertainties given in Equation (1).

Xie [20] provides the following useful lemma:

Lemma 1. Given matrices $Q=Q^{T}, H, E$ and $R=R^{T}>0$ of appropriate dimensions,

$$
Q+H F E+E^{T} F^{T} H^{T}<0
$$

for all $F$ satisfying $F^{T} F \leq R$, if and only if there exists some $\lambda>0$ such that

$$
Q+\lambda H H^{T}+\lambda^{-1} E^{T} R E<0 .
$$

Theorem 2. For given scalars $h>0, \tau>0$, and $\mu$, the neutral system given in Equation (1) is robustly stable for any delay $\tau(t)$ satisfying Condition (2) if the operator $G$ is stable, and there exist positive definite matrices $P>0, P_{1}>0, Q_{1}>0, Q_{2}>0, Q_{3}>0, R_{k}>0, S_{k}>0$, $Z_{k}>0, k=1,2$, and appropriately dimensioned matrices $T_{i}, N_{i}, Y_{i}, W_{i}, i=1, \ldots, 6$ such that the following linear matrix inequality (LMI) holds:

$$
\left[\begin{array}{ccccccccccccc}
\hat{\Omega}_{11} & \hat{\Omega}_{12} & \hat{\Omega}_{13} & \hat{\Omega}_{14} & \hat{\Omega}_{15} & \hat{\Omega}_{16} & -\tau T_{1} & -\tau N_{1} & -h Y_{1} & -h W_{1} & A^{T} M & A^{T} S & \left(P+P_{1}\right) D \\
* & \hat{\Omega}_{22} & \hat{\Omega}_{23} & \hat{\Omega}_{24} & \hat{\Omega}_{25} & \hat{\Omega}_{26} & -\tau T_{2} & -\tau N_{2} & -h Y_{2} & -h W_{2} & A_{1}^{T} M & A_{1}^{T} S & 0 \\
* & * & \hat{\Omega}_{33} & \hat{\Omega}_{34} & \hat{\Omega}_{35} & \hat{\Omega}_{36} & -\tau T_{3} & -\tau N_{3} & -h Y_{3} & -h W_{3} & 0 & 0 & 0 \\
* & * & * & \hat{\Omega}_{44} & \hat{\Omega}_{45} & \hat{\Omega}_{46} & -\tau T_{4} & -\tau N_{4} & -h Y_{4} & -h W_{4} & 0 & 0 & -C^{T} P_{1} D \\
* & * & * & * & \hat{\Omega}_{55} & \hat{\Omega}_{56} & -\tau T_{5} & -\tau N_{5} & -h Y_{5} & -h W_{5} & 0 & 0 & 0 \\
* & * & * & * & * & \hat{\Omega}_{66} & -\tau T_{6} & -\tau N_{6} & -h Y_{6} & -h W_{6} & 0 & C^{T} S & 0 \\
* & * & * & * & * & * & -\tau R_{1} & 0 & 0 & 0 & 0 & 0 & 0 \\
* & * & * & * & * & * & * & -\tau R_{2} & 0 & 0 & 0 & 0 & 0 \\
* & * & * & * & * & * & * & * & -h Z_{1} & 0 & 0 & 0 & 0 \\
* & * & * & * & * & * & * & * & * & -h Z_{2} & 0 & 0 & 0 \\
* & * & * & * & * & * & * & * & * & * & -M & 0 & M D \\
* & * & * & * & * & * & * & * & * & * & * & -S & S D \\
* & * & * & * & * & * & * & * & * & * & * & * & -I
\end{array}\right]
$$

where

$$
\begin{aligned}
\hat{\Omega}_{11}= & P A+A^{T} P+P_{1} A+A^{T} P_{1}+Q_{1}+S_{1}+S_{2}+ \\
& Y_{1}+Y_{1}^{T}+T_{1}+T_{1}^{T}+W_{1}+W_{1}^{T}+N_{1}+N_{1}^{T}+E^{T} E, \\
\hat{\Omega}_{12}= & P A_{1}+P_{1} A_{1}+Y_{2}^{T}-T_{1}+T_{2}^{T}+W_{2}^{T}-N_{1}+N_{2}^{T}+E^{T} E_{1}, \\
\hat{\Omega}_{13}= & Y_{3}^{T}+T_{1} C+T_{3}^{T}+W_{3}^{T}+N_{3}^{T}, \\
\hat{\Omega}_{14}= & -A^{T} P_{1} C-Y_{1}(C+I)+Y_{4}^{T}-T_{1} C+T_{4}^{T}-W_{1}+W_{4}^{T}+N_{4}^{T}, \\
\hat{\Omega}_{15}= & Y_{1} C+Y_{5}^{T}+T_{5}^{T}+W_{5}^{T}+N_{5}^{T}, \\
\hat{\Omega}_{16}= & P C+Y_{6}^{T}+T_{6}^{T}+W_{6}^{T}+N_{6}^{T}, \\
\hat{\Omega}_{22}= & -(1-\mu) S_{1}-T_{2}-T_{2}^{T}-N_{2}-N_{2}^{T}+E_{1}^{T} E_{1},
\end{aligned}
$$




$$
\begin{aligned}
\hat{\Omega}_{23} & =T_{2} C-T_{3}^{T}-N_{3}^{T}, \\
\hat{\Omega}_{24} & =-A_{1}^{T} P_{1} C-Y_{2}(C+I)-T_{2} C-T_{4}^{T}-W_{2}-N_{4}^{T}, \\
\hat{\Omega}_{25} & =Y_{2} C-T_{5}^{T}-N_{5}^{T}, \\
\hat{\Omega}_{26} & =-T_{6}^{T}-N_{6}^{T}, \\
\hat{\Omega}_{33} & =-(1-\mu) S_{2}+T_{3} C+C^{T} T_{3}^{T}, \\
\hat{\Omega}_{34} & =-Y_{3}(C+I)-T_{3} C+C^{T} T_{4}^{T}-W_{3}, \\
\hat{\Omega}_{35} & =Y_{3} C+C^{T} T_{5}^{T}, \\
\hat{\Omega}_{36} & =C^{T} T_{6}^{T}, \\
\hat{\Omega}_{44} & =-Q_{1}+Q_{2}-Y_{4}(C+I)-(C+I)^{T} Y_{4}^{T}-T_{4} C-C^{T} T_{4}^{T}-W_{4}-W_{4}^{T}, \\
\hat{\Omega}_{45} & =Y_{4} C-(C+I)^{T} Y_{5}^{T}-C^{T} T_{5}^{T}-W_{5}^{T}, \\
\hat{\Omega}_{46} & =-(C+I)^{T} Y_{6}^{T}-C^{T} T_{6}^{T}-W_{6}^{T}, \\
\hat{\Omega}_{55} & =-Q_{2}+Y_{5} C+C^{T} Y_{5}^{T}, \\
\hat{\Omega}_{56} & =C^{T} Y_{6}^{T}, \\
\hat{\Omega}_{66} & =-Q_{3}, \\
M & =h Z_{1}+\tau R_{1}, \\
S & =Q_{3}+h Z_{2}+\tau R_{2} .
\end{aligned}
$$

Proof. If $A$ and $A_{1}$ in Inequality (8) in Theorem 1 are replaced by $A+D F(t) E$ and $A_{1}+D F(t) E_{1}$ respectively, then Inequality (8) for the uncertain system given in Equation (1) is equivalent to the following condition:

$$
\tilde{\Omega}+\Gamma_{d} F(t) \Gamma_{e}^{T}+\Gamma_{e} F^{T}(t) \Gamma_{d}^{T}<0,
$$

where

$$
\begin{aligned}
& \Gamma_{d}^{T}=\left[D^{T}\left(P+P_{1}\right), 0,0,-D^{T} P_{1} C, 0,0,0,0,0,0, D^{T} M, D^{T} S\right], \\
& \Gamma_{e}=\left[E, E_{1}, 0,0,0,0,0,0,0,0,0,0\right]^{T} .
\end{aligned}
$$

By Lemma 1, a necessary and sufficient condition to satisfy Inequality (53) for the system given in Equation (1) is that there exists a $\lambda>0$ such that

$$
\tilde{\Omega}+\lambda \Gamma_{d} \Gamma_{d}^{T}+\lambda^{-1} \Gamma_{e}^{T} \Gamma_{e}<0 .
$$

Multiplying both sides of Equation (54) by $\lambda$, replacing $\lambda P, \lambda P_{1}, \lambda Q_{1}, \lambda Q_{2}, \lambda Q_{3}, \lambda Z_{1}, \lambda Z_{2}, \lambda R_{1}$, $\lambda R_{2}, \lambda S_{1}, \lambda S_{2}, \lambda T_{i}, \lambda Y_{i}, \lambda N_{i}$, and $\lambda W_{i}$ by $P, P_{1}, Q_{1}, Q_{2}, Q_{3}, Z_{1}, Z_{2}, R_{1}, R_{2}, S_{1}, S_{2}, T_{i}, Y_{i}, N_{i}$, and $W_{i}$ for $i=1, \ldots, 6$, and applying Schur complement, we find Inequality (54) is equivalent to Inequality (52). This completes the proof of Theorem 2.

\section{Numerical examples}

In this Section, we provide two examples as a demonstration that the methods presented in this paper are effective and are an improvement over existing methods.

Example 1. Consider the following neutral system with time-varying discrete delay:

$$
\dot{x}(t)-\left[\begin{array}{cc}
-0.2 & 0 \\
0.2 & -0.1
\end{array}\right] \dot{x}(t-h)=\left[\begin{array}{cc}
-0.9 & 0.2 \\
0.1 & -0.9
\end{array}\right] x(t)+\left[\begin{array}{cc}
-1.1 & -0.2 \\
-0.1 & -1.1
\end{array}\right] x(t-\tau(t)) .
$$

Let the state delay $\tau(t)$ be time-varying with derivative $\mu=0.01$; in this example we use a delay with fixed derivative. Table 1 shows the maximum allowable state delays which guarantee stability of this system as $h$ varies from 0.1 to 1.7076, computed using Theorem 1. Note that the stability criterion in [9] can not be applied to this example since it is only applicable to systems with a constant state delay $\tau$, while here the state delay $\tau(t)$ is a function of $t$. Clearly, in this sense, our 
Table 1: Allowable time delay $\tau(t)$ from Theorem 1 for Example 1 with $\mu=0.01$

\begin{tabular}{|c|c|c|c|c|c|c|c|c|c|}
\hline$h$ & 0.1 & 0.2 & 0.3 & 0.4 & 0.5 & 0.6 & 0.7 & 0.8 & 0.9 \\
\hline$\overline{\tau(t)}$ & 1.7728 & 1.7641 & 1.7552 & 1.7464 & 1.7378 & 1.7296 & 1.7221 & 1.7156 & 1.7110 \\
\hline$h$ & 1.0 & 1.1 & 1.2 & 1.3 & 1.4 & 1.5 & 1.6 & 1.7076 & 10000 \\
\hline$\tau(t)$ & 1.7086 & 1.7078 & 1.7076 & 1.7076 & 1.7076 & 1.7076 & 1.7076 & 1.7076 & 1.7076 \\
\hline
\end{tabular}

Table 2: Allowable time delay $\tau_{1}$ for Example 1 with $\tau(t)=\tau_{1}$ and $h=\tau_{2}$

\begin{tabular}{|c|c|c|c|c|c|c|c|c|c|c|}
\hline Method & $\overline{\tau_{2}}$ & 0.1 & 0.2 & 0.3 & 0.4 & 0.5 & 0.6 & 0.7 & 0.8 & 0.9 \\
\hline He et al & $\tau_{1}$ & 1.7100 & 1.6987 & 1.6883 & 1.6792 & 1.6718 & 1.6664 & 1.6624 & 1.6591 & 1.6564 \\
\hline Ours & $\tau_{1}$ & 1.7844 & 1.7757 & 1.7669 & 1.7581 & 1.7495 & 1.7413 & 1.7338 & 1.7273 & 1.7226 \\
\hline Method & $\tau_{2}$ & 1.0 & 1.1 & 1.2 & 1.3 & 1.4 & 1.5 & 1.6 & 1.6527 & 10000 \\
\hline He et al & $\tau_{1}$ & 1.6543 & 1.6531 & 1.6527 & 1.6527 & 1.6527 & 1.6527 & 1.6527 & 1.6527 & 1.6527 \\
\hline Ours & $\tau_{1}$ & 1.7201 & 1.7193 & 1.7191 & 1.7191 & 1.7191 & 1.7191 & 1.7191 & 1.7191 & 1.7191 \\
\hline
\end{tabular}

criterion is an improvement over that in [9]. When $\mu=0.01$ and $h=0.1$, the allowable maximum time delay $\tau(t)$ is 1.7728. For $\mu=0.01$, the maximum allowable delay $\tau(t)$ is approximately 1.7076 when $h$ is in the range 1.2 to 1000 . Initially, the allowable time delay $\tau(t)$ decreases as the neutral delay $h$ increases, for small $h$, but the allowable time delay $\tau(t)$ remains almost unchanged when $h \geq 1.2$.

Chen [1], Fridman [2], Lien et al [13] and He et al [9] studied the above neutral system in the case when $\tau(t)=\tau_{1}, h=\tau_{2}, \mu=0$ and $\tau_{1}=\tau_{2}$. The upper bounds on delays under which it is possible to guarantee the stability of this system using the methods in [13, 1, 2, 9] are $\tau_{1}=\tau_{2}=0.3$, $\tau_{1}=\tau_{2}=0.5658, \tau_{1}=\tau_{2}=0.74$ and $\tau_{1}=\tau_{2}=1.6527$, respectively. In contrast, by solving the matrix inequality (8) in Theorem 1 of this paper for $\tau_{1}=\tau_{2}$, we obtain maximum upper bounds on the allowable delay of $\tau_{1}=\tau_{2}=1.7191$, which are greater than those obtained by any of the aforementioned methods, demonstrating the superiority of our approach.

In the particular case when $\tau(t)=\tau_{1}, h=\tau_{2}$ and $\tau_{1} \neq \tau_{2}$, Table 2 lists the upper bounds on $\tau_{1}$ for which stability can be guaranteed, for various values of $\tau_{2}$ from 0.1 to 10000 , using the methods in this paper and the approach in [9]. It can be seen that our delay-dependent stability criterion is considerably less conservative than that in [9]. Note again that the upper bound on $\tau_{1}$ decreases as $\tau_{2}$ increases when $\tau_{2}$ is small, but the upper bound on $\tau_{1}$ remains almost unchanged when $\tau_{2} \geq 1.2$.

Remark 4. When $h=\tau_{2}=0.1$ and $\mu=0$ in Example 1, He et al [9] obtained the maximum upper bound $\tau_{1}=1.7100$ for which stability of the system in Example 1 can be guaranteed. Theorem 1 in this paper gives a maximum upper bound on the allowable delay of $\tau_{1}=1.7844$. Let us consider the contribution of the additional matrices in Theorem 1 for this particular case, i.e. $h=\tau_{2}=0.1$ and $\mu=0$. If we set $N_{3}=N_{4}=N_{5}=N_{6}=T_{3}=T_{5}=T_{6}=W_{2}=W_{3}=W_{5}=W_{6}=Y_{1}=$ $Y_{2}=Y_{3}=Y_{4}=Y_{6}=0$ and replace $Q_{2}$ by 0 in Matrix Inequality (8) in Theorem 1 of this paper, we obtain a solution to Matrix Inequality (8), giving the maximum upper bound on the allowable delay of $\tau_{1}=1.7844$. Matrices $P, P_{1}, Q_{1}, Q_{3}, R_{1}, R_{2}, S_{1}, S_{2}, Z_{1}, Z_{2}, N_{1}, N_{2}, T_{1}, T_{2}, T_{4}, Y_{5}$, $W_{1}$, and $W_{4}$ contribute to this allowable maximum time delay. Compared to the result in [9], the additional matrices $P, R_{1}, S_{2}, Z_{1}, T_{1}, T_{2}, T_{4}$, and $Y_{5}$ in Matrix Inequality (8) contribute to the improvement of the allowable maximum time delay $\tau_{1}$. 
Table 3: Allowable time delay $\tau_{1}$ for Example 2 with $\tau(t)=\tau_{1}$ and $h=\tau_{2}$

\begin{tabular}{lllllllllll}
\hline Method & $c$ & 0 & 0.05 & 0.1 & 0.15 & 0.2 & 0.25 & 0.3 & 0.35 & 0.4 \\
\hline Han [6] & $\tau_{2}=\tau_{1}$ & 1.77 & 1.63 & 1.48 & 1.33 & 1.16 & 0.98 & 0.79 & 0.59 & 0.37 \\
He et al & $\tau_{2}=\tau_{1}$ & 2.39 & 2.05 & 1.75 & 1.49 & 1.27 & 1.08 & 0.91 & 0.76 & 0.63 \\
Theorem 2 & $\tau_{2}=\tau_{1}$ & 2.39 & 2.13 & 1.89 & 1.67 & 1.48 & 1.30 & 1.15 & 1.00 & 0.87 \\
\hline He et al & $\tau_{2}=10000$ & 2.39 & 2.05 & 1.75 & 1.49 & 1.27 & 1.08 & 0.91 & 0.76 & 0.63 \\
Theorem 2 & $\tau_{2}=10000$ & 2.39 & 2.13 & 1.89 & 1.67 & 1.48 & 1.30 & 1.15 & 1.00 & 0.87 \\
\hline He et al & $\tau_{2}=0.1$ & 2.39 & 2.25 & 2.11 & 1.96 & 1.81 & 1.66 & 1.50 & 1.33 & 1.16 \\
Theorem 2 & $\tau_{2}=0.1$ & 2.39 & 2.25 & 2.11 & 1.96 & 1.82 & 1.66 & 1.52 & 1.34 & 1.18 \\
\hline
\end{tabular}

Remark 5. Next, we illustrate the contribution of the additional matrices $P$ and $T_{1}$ when $h=$ $\tau_{2}=0.1$ and $\mu=0$ in Example 1 .

1) If $P$ is replaced by 0 in Matrix Inequality (8), then the allowable maximum time delay $\tau_{1}$ under which the neutral system given in Example 1 is asymptotically stable decreases from 1.7844 to 1.7802. Thus, the additional matrix $P$ in Theorem 1 clearly contributes to the improvement in the allowable time delay.

2) If we set $N_{5}=N_{3}=T_{6}=T_{5}=T_{4}=T_{3}=T_{2}=W_{5}=W_{3}=Y_{6}=Y_{5}=Y_{4}=Y_{3}=Y_{2}=Y_{1}=0$ and $P$ is replaced by 0 in Matrix Inequality (8), the allowable maximum time delay $\tau_{1}$ under which the neutral system given in Example 1 is asymptotically stable remains the same at 1.7802. If we further set $T_{1}=0$, then the allowable maximum time delay $\tau_{1}$ decreases from 1.7802 to 1.7100. Thus, the additional matrix $T_{1}$ in Theorem 1 clearly contributes to the improvement in the allowable time delay.

Example 2. Consider the robust stability of the neutral system in the form given in Equation (1) which is associated with the following nominal system:

$$
\dot{x}(t)-\left[\begin{array}{cc}
c & 0 \\
0 & c
\end{array}\right] \dot{x}(t-h)=\left[\begin{array}{cc}
-2 & 0 \\
0 & 0.9
\end{array}\right] x(t)+\left[\begin{array}{cc}
-1 & 0 \\
-1 & -1
\end{array}\right] x(t-\tau(t)),
$$

where $D=I$, and $E=E_{1}=0.2 I$. Again, no conclusions can be made using the stability criteria in [9]. We only consider the special case $h=\tau_{2}, \tau(t)=\tau_{1}$. The upper bounds on the delay $\tau_{1}$ under which robust stability of this system can be guaranteed using the methods in [6, 9] and Theorem 2 of this paper are listed in Table 3, as c varies from 0 to 0.4 . For $\tau_{1}=\tau_{2}$, it is clear that our results are significantly better than those in [6,9] because our allowable maximum time delay $\tau_{1}$ is larger. For $\tau_{1} \neq \tau_{2}$ Han's method [6] is inapplicable; setting $\tau_{2}=10000$, again our results are much better than those provided by the approach in [9]. For $\tau_{1} \neq \tau_{2}$ and $\tau_{2}=0.1$, our results are slightly better than those in [9]. Overall, again these results demonstrate that the delay-dependent robust stability condition in our paper is less conservative than the existing approaches in [6, 9].

Remark 6. He et al [9] obtained the allowable maximum time delay $\tau_{1}=0.63$ for which the robust stability of the system given in Example 2 is guaranteed, with settings $\tau(t)=\tau_{1}, h=\tau_{2}$, $\tau_{1}=\tau_{2}$ and $c=0.4$. However, the upper bound on the delay $\tau_{1}$ under which robust stability of this system can be guaranteed using Theorem 2 of this paper is 0.87 when $c=0.4$. With the same settings, let us now set $N_{3}=N_{4}=N_{5}=N_{6}=T_{1}=T_{2}=T_{3}=T_{4}=T_{5}=T_{6}=W_{1}=W_{2}=$ $W_{3}=W_{5}=W_{6}=Y_{1}=Y_{2}=Y_{3}=Y_{4}=Y_{6}=0$ and replace matrices $Q_{2}$ and $R_{1}$ by zero matrices in Theorem 2. Since $R_{1}$ is replaced by 0, Matrix Inequality (52) has to be modified. Noting that $\mu=0$, we solve the modified Matrix Inequality (52). The allowable maximum time delay under which robust stability of this system can be guaranteed is still $\tau_{1}=0.87$. Matrices $P, Q_{1}, Q_{3}, R_{2}$, $S_{1}, S_{2}, Z_{1}, Z_{2}, P_{1}, N_{1}, N_{2}, Y_{5}$ in Matrix Inequality (52) contribute to this allowable maximum time delay $\tau_{1}=0.87$. Therefore, compared with the result in [9], the additional matrices $P, S_{2}, Z_{1}$, and $Y_{5}$ in Theorem 2 contribute to the improvement of the allowable time delay in this example. 


\section{Conclusion}

New delay-dependent stability criteria for neutral systems with time-varying discrete delay and time-varying structured uncertainties have been obtained. These criteria are dependent on the neutral-delay, the discrete-delay and the derivative of the discrete-delay, and as a result, our approach reduces the conservatism present in existing methods. Numerical examples have been given to demonstrate that results derived using the criteria in this paper are less conservative than various existing ones in the literature.

\section{References}

[1] J.D. Chen, C.H. Lien, K.K. Fan, J.H. Chou, Criteria for asymptotic stability of a class of neutral systems via a LMI approach, IEE Pro. Control Theory Appl. 148 (2001) 442-447.

[2] E. Fridman, New Lyapunov-Krasovaskii functionals for stability of linear retarded and neutral type systems, Systems Control Lett. 43 (2001) 309-319.

[3] E. Fridman, U. Shaked, An improved stabilization method for linear time-delay systems, IEEE Trans. Automat. Control 47 (2002) 1931-1937.

[4] E. Fridman, U. Shaked, Delay-dependent stability and $H_{\infty}$ control: constant and time-varying delays, Int. J. Control 76 (2003) 48-60.

[5] Q.L. Han, On delay-dependent stability for neutral delay-differential systems, Int. J. Appl. Math. Comput. Sci. 11 (2001) 965-976.

[6] Q.L. Han, Robust stability of uncertain delay-differential systems of neutral type, Automatica 38 (2002) 719-723.

[7] Q.L. Han, On stability of linear neutral systems with mixed time delays: A discretized Lyapunov functional approach, Automatica 41 (2005) 1209-1218.

[8] S. Hara, Y. Yamamoto, T. Omata, M. Nakano, Repetitive control system: a new type servo system for periodic exogenous signals, IEEE Trans. Automat. Control 33 (1988), 659-668.

[9] Y. He, M. Wu, J.H. She, G.P. Liu, Delay-dependent robust stability criteria for uncertain neutral systems with mixed delays, Systems Control Lett. 51 (2004) 57-65.

[10] D. Ivănescu, S.I. Niculescu, L. Dugard, J.M. Kion, E.I. Verriest, On delay-dependent stability for linear neutral systems, Automatica 39 (2003) 255-261.

[11] J.H. Kim, Delay and its time-derivative dependent robust stability of time-delayed linear systems with uncertainty, IEEE Trans. Automat. Control 46 (2001), 789-792.

[12] C.H. Lien, New stability criterion for a class of uncertain nonlinear neutral time-delay systems, Int. J. Systems. Sci. 32 (2001) 20-27.

[13] C.H. Lien, K.W. Yu, J.G. Hsieh, Stability conditions for a class of neutral systems with multiple delays, J. Math. Anal Appl. 245 (2000) 20-27.

[14] Y.S. Moon, P.G. Park, W.H. Kwon, Y.S. Lee, Delay-dependent robust stabilization of uncertain state-delay systems, Int. J. Control 74 (2001) 1447-1455.

[15] S.I. Niculescu, On delay-dependent stability under model transformations of some neutral linear systems, Int. J. Control 74 (2001) 609-617.

[16] J.H. Park, A new delay-dependent criterion for neutral systems with multiple delays, J. Comput. Appl. Math. 136 (2001) 177-184. 
[17] J.H. Park, Stability criterion for neutral differential systems with mixed multiple time-varying delay arguments, Math. Comput. Simulation 59 (2002) 401-412.

[18] P.G. Park, A delay-dependent stability criterion for systems with uncertain time-invariant delays, IEEE Trans. Automat. Control, 44 (1999) 876-877.

[19] M. Wu, Y. He, J.H. She, G.P. Liu, Delay-dependent criteria for robust stability of time-varying delay systems, Automatica 40 (2004) 1435-1439.

[20] L. Xie, Output feedback $H_{\infty}$ control of system with parameter uncertainty, Int. J. Control 63 (1996) $741-750$.

[21] S.Y. Xu, J. Lam, Improved delay-dependent stability criteria for time-delay system, IEEE Trans. Automat. Control 50 (2005) 384-387.

[22] D. Yue, S. Won, An improvement on 'Delay and its time-derivative dependent robust stability of time-delayed linear system with uncertainty, IEEE Trans. Automat. Control, 47 (2002) 407-408. 